\title{
A New Colorless and Transparent Polyetherimide Derived from A Bio-based Plant Oil (Anethole): Synthesis and Properties
}

Yuanqiang Wang, Junfeng Zhou, Xiaoyao Chen, Jing Sun* and Qiang Fang*

*Q. Fang. E-mail: qiangfang@mail.sioc.ac.cn; *J. Sun. Email: sunjing@sioc.ac.cn

Key Laboratory of Synthetic and Self-Assembly Chemistry for Organic Functional

Molecules, Center for Excellence in Molecular Synthesis, Shanghai Institute of Organic Chemistry, University of Chinese Academy of Sciences, Chinese Academy of Sciences, 345 Lingling Road, Shanghai 200032, PR China.

\begin{tabular}{|c|c|}
\hline Numbers of Pages & 5 (S1 to S5) \\
\hline Numbers of Figures & 9 (Figure S1 to S9) \\
\hline Numbers of Tables & 1 (Table S1) \\
\hline
\end{tabular}




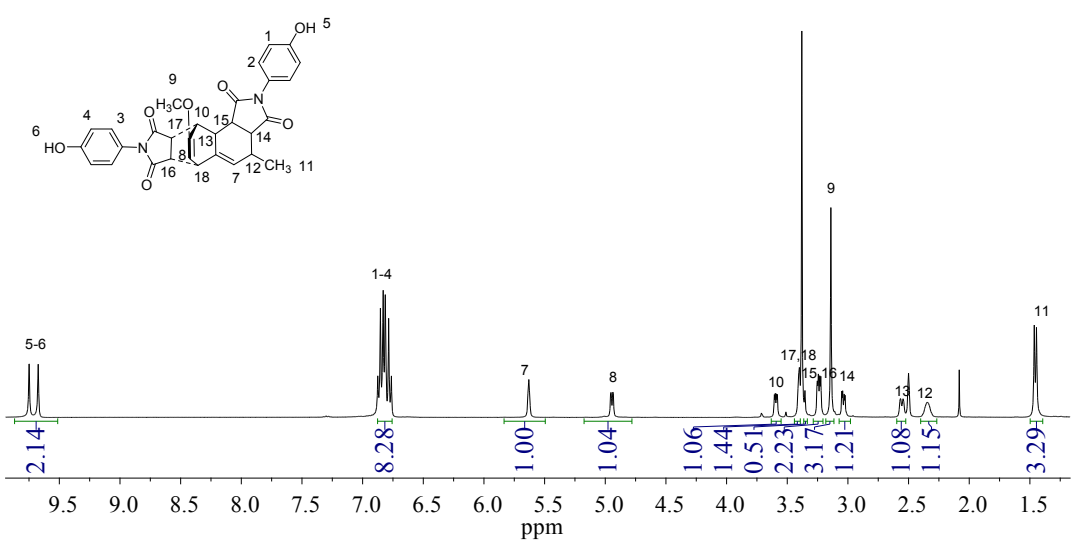

Figure. S1 The ${ }^{1} \mathrm{H}$ NMR spectrum of compound 1 (400 MHz, $d_{6}$-DMSO).
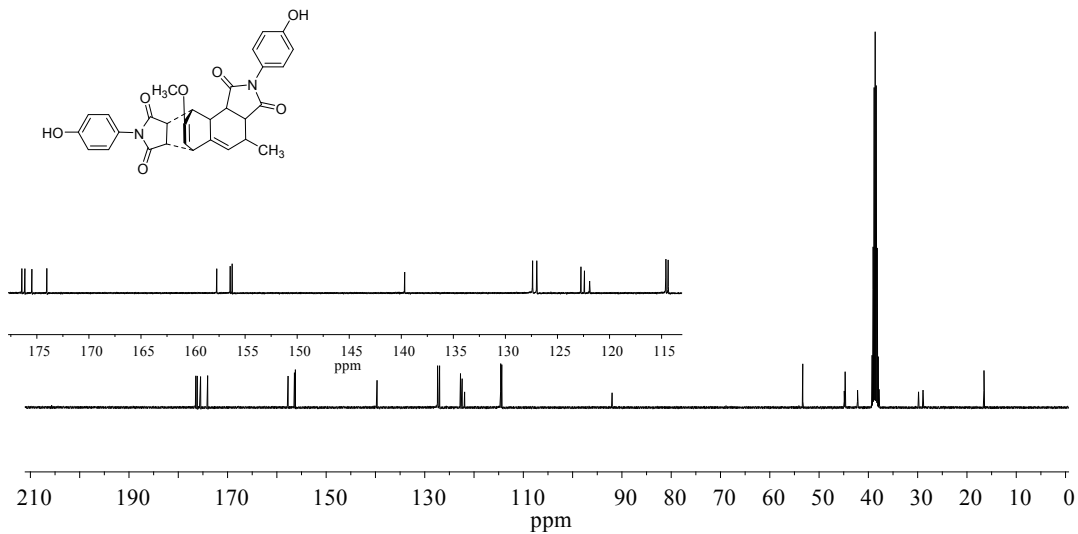

Figure. S2 The ${ }^{13} \mathrm{C}$ NMR spectrum of compound 1 (101 MHz, $d_{6}$-DMSO).

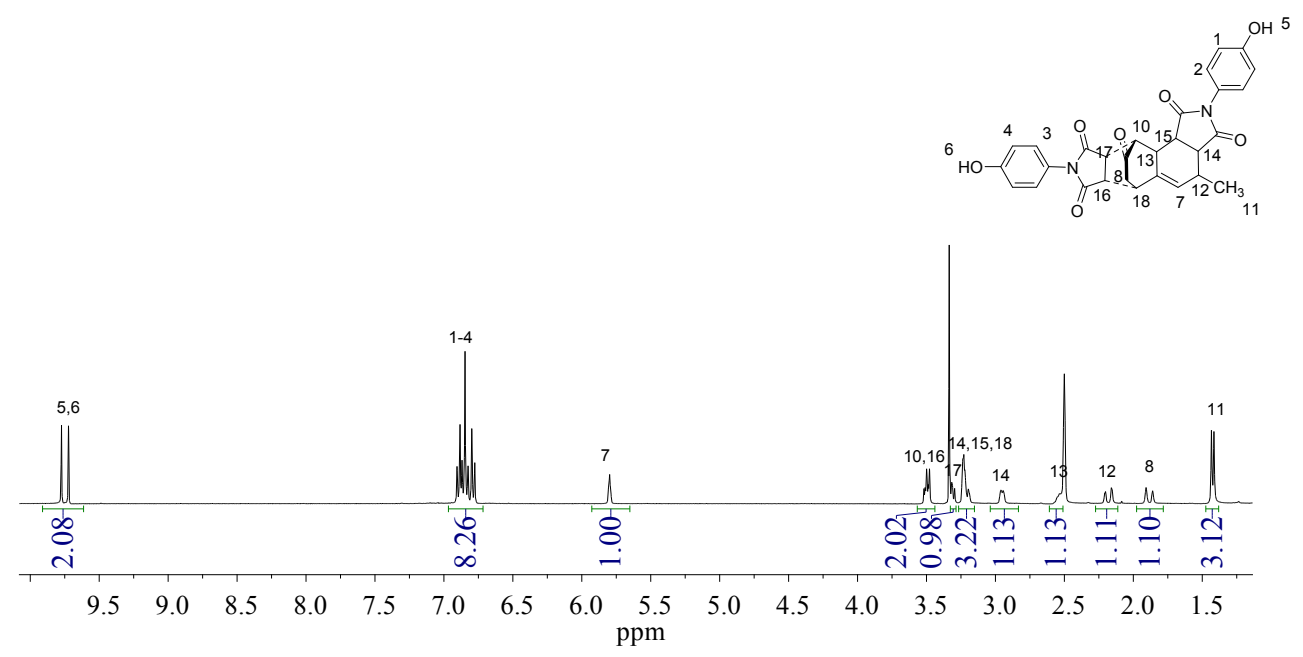

Figure. S3 The ${ }^{1} \mathrm{H}$ NMR spectrum of compound 2 (400 MHz, $d_{6}$-DMSO). 


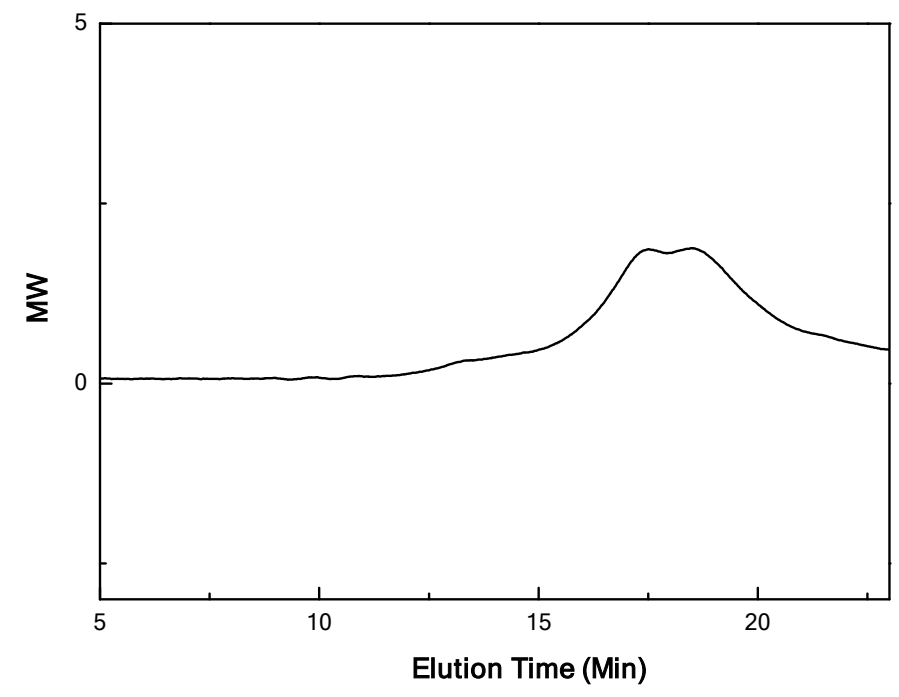

Figure. S7 The GPC curve of PI-I.

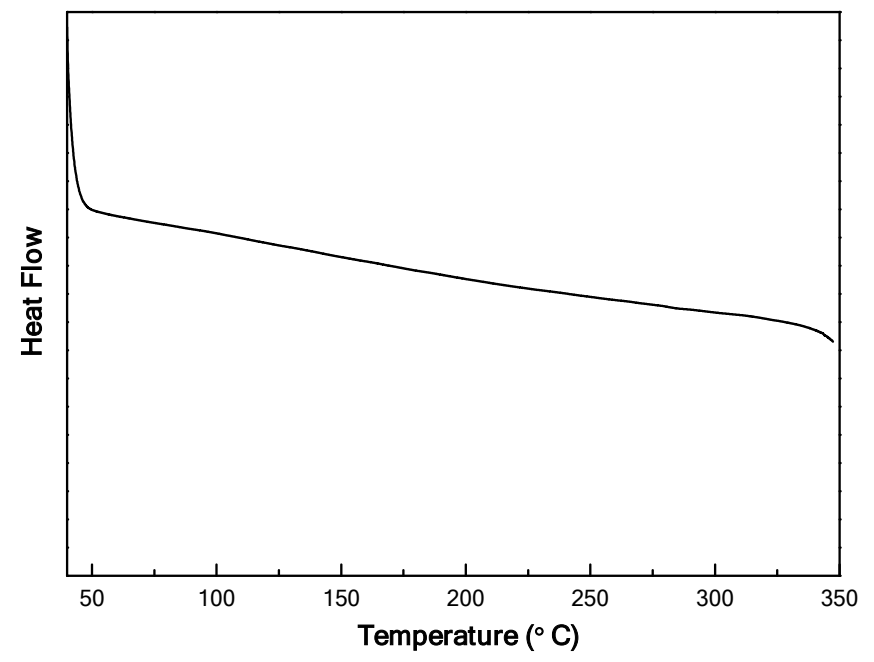

Figure. S8 DSC curve of the PI-I film $\left(10^{\circ} \mathrm{C} / \mathrm{min}\right.$, in nitrogen $)$.

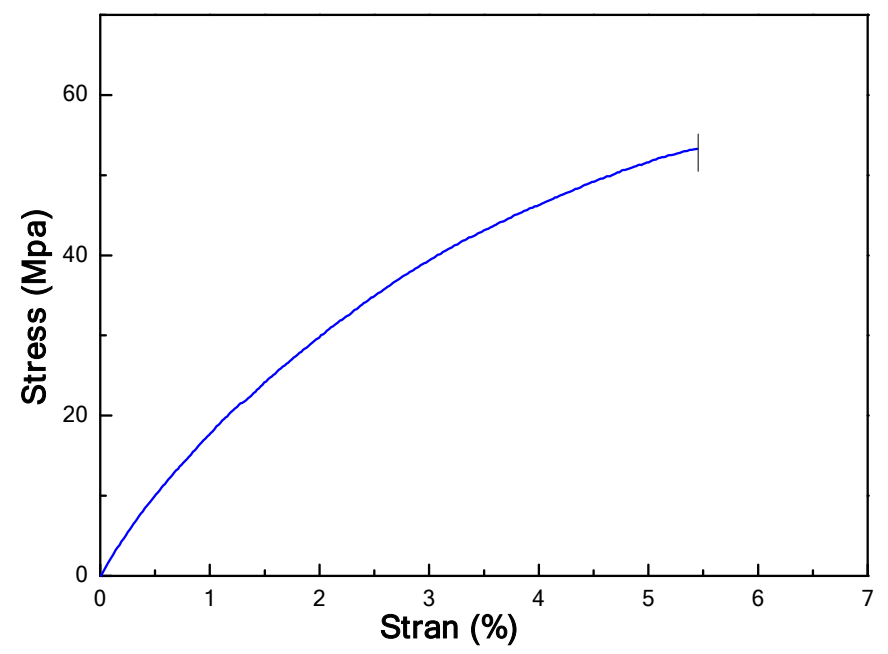

Fig. S9 Stress-strain curves of PI-I film. 
Table S1. Crystal data and structure refinement for compound 1 with one molecular $\mathrm{H}_{2} \mathrm{O}$.

\begin{tabular}{|c|c|c|}
\hline Identification code & \multicolumn{2}{|c|}{ Compound 2 with one molecular $\mathrm{H}_{2} \mathrm{O}$} \\
\hline Empirical formula & \multicolumn{2}{|l|}{$\mathrm{C}_{30} \mathrm{H}_{28} \mathrm{~N}_{2} \mathrm{O}_{8}$} \\
\hline Crystal size & \multicolumn{2}{|c|}{$0.180 \times 0.140 \times 0.110 \mathrm{~mm}^{3}$} \\
\hline Formula weight & \multicolumn{2}{|l|}{544.54} \\
\hline Crystal system & \multicolumn{2}{|l|}{ Monoclinic } \\
\hline Space group & \multicolumn{2}{|l|}{$\mathrm{P} 21 / \mathrm{c}$} \\
\hline \multirow[t]{3}{*}{ Unit cell dimensions } & $\mathrm{a}=15.4776(10) \AA$ & $\alpha=90^{\circ}$. \\
\hline & $\mathrm{b}=9.9787(6) \AA$ & $\beta=114.023(2)^{\circ}$. \\
\hline & $\mathrm{c}=18.9692(15) \AA$ & $\gamma=90^{\circ}$. \\
\hline Volume & \multicolumn{2}{|l|}{$2676.0(3) \AA^{3}$} \\
\hline $\mathrm{Z}$ & \multicolumn{2}{|l|}{4} \\
\hline Density (calculated) & \multicolumn{2}{|l|}{$1.352 \mathrm{mg} / \mathrm{cm}^{3}$} \\
\hline $\mathrm{F}(000)$ & \multicolumn{2}{|l|}{1144} \\
\hline Theta range for data collection & \multicolumn{2}{|l|}{2.355 to $25.994^{\circ}$. } \\
\hline Independent reflections & \multicolumn{2}{|c|}{$5232[\mathrm{R}(\mathrm{int})=0.0389]$} \\
\hline Absorption correction & \multicolumn{2}{|c|}{ Semi-empirical from equivalents } \\
\hline Refinement method & \multicolumn{2}{|c|}{ Full-matrix least-squares on $\mathrm{F}^{2}$} \\
\hline Data / restraints / parameters & \multicolumn{2}{|l|}{$5232 / 0 / 369$} \\
\hline Goodness-of-fit on $\mathrm{F}^{2}$ & \multicolumn{2}{|l|}{1.032} \\
\hline Final $R$ indices $[\mathrm{I}>2 \operatorname{sigma}(\mathrm{I})]$ & \multicolumn{2}{|c|}{$\mathrm{R} 1=0.0570, \mathrm{wR} 2=0.1390$} \\
\hline $\mathrm{R}$ indices (all data) & \multicolumn{2}{|c|}{$\mathrm{R} 1=0.0940, \mathrm{wR} 2=0.1677$} \\
\hline
\end{tabular}

